\title{
Co-medication with Cannabidiol May Slow Down the Progression of Motor Neuron Disease: A Case Report
}

\section{Gerhard Nahler*}

CIS Clinical Investigation Support $\mathrm{GmbH}$, Wien, Austria

*Corresponding author: Gerhard Nahler, CIS Clinical Investigation Support GmbH, Wien, Austria, Tel: +43-1-5234015; E-mail: cis-qa@aon.at

Received date: July 10, 2017; Accepted date: July 12, 2017; Published date: July 15, 2017

Copyright: (C) 2017 Nahler G, et al. This is an open-access article distributed under the terms of the Creative Commons Attribution License, which permits unrestricted use, distribution, and reproduction in any medium, provided the original author and source are credited.

\begin{abstract}
Amyotrophic lateral sclerosis (ALS, also called Charcot disease, Lou Gehrig disease), is a progressive, neurodegenerative disease caused by the degeneration of motor neurons in the brain and spinal cord. There is no cure. This report describes a case of motor neuron disease with typical weakness in one leg, one hand and the tongue. Despite of treatment with riluzole, symptoms progressed relatively fast. Therefore, the patient decided to take cannabidiol (CBD, $2 \times 200 \mathrm{mg} /$ day) as co-medication, which was started 8 weeks after riluzole, and increased to a daily dose of $2 \times 300 \mathrm{mg}$. Within 6 weeks, the impaired function of the right hand and foot reversed almost completely and dysphagia partially. Improvement was maintained for about 10 weeks, when again a slow progression of dysarthria and dysphagia was observed. Eighteen months after onset, speech is almost completely lost, and dysphagia also progressed. However, symptoms of the limbs (weakness, fasciculation, atrophy) worsened much less. It is concluded, that Co-medication with CBD may be able to slow down the progression of some but not all symptoms of motor neuron disease.
\end{abstract}

Keywords: Amyotrophic lateral sclerosis; Cannabidiol; Cannabinoids; Motor neuron disease

Abbreviations ALS: Amyotrophic Lateral Sclerosis; CBD: Cannabidiol; CB1: Cannabinoid Receptor 1; CB2: Cannabinoid Receptor 2; COX: Cyclooxigenase; GPR: G-protein coupled Receptor; IL: Interleukine; MND: Motor Neuron Disease; PPARg: Peroxisome Proliferator Activated Receptor gamma; THC: Delta-9tetrahydrocannabinol; TNF-a: Tumor Necrosis Factor Alpha

\section{Introduction}

Amyotrophic lateral sclerosis, also called maladie de Charcot/ Charcot disease or Lou Gehrig disease, is a rare neurodegenerative disorder characterized by progressive degeneration of motor neurons in the brain and spinal cord with an incidence of about 1 to 3 cases/ 100,000 [1]. It affects most often middle aged adults, men slightly more often than women.

Although ALS is clinically a heterogeneous disease, the first sign of ALS is often weakness in one leg, one hand, the face, or the tongue. The weakness slowly spreads to both arms and both legs. Over time, problems with speaking, eating, walking, and breathing increase. There is no cure. Weight loss is nearly ubiquitous in ALS and has a negative, statistically significant impact on survival [2]. The most common causes of death are pneumonia, lung- or heart failure. Respiratory failure occurs within three to five years of the onset of symptoms, though about $10 \%$ of sufferers live for 10 or more years. Because of the variability in the speed of the progression, median survival varies widely between 676 days [3] and 67 to 71 months [4,5]. As ALSphenotypes differ, each person's disease course is likely unique.

The cause of ALS is unknown; in about 5 to $10 \%$ of the cases there is a positive family history. Some of these cases seem to be associated with mutations in the gene for an enzyme called copper-zinc superoxide dismutase $1(\mathrm{Cu}-\mathrm{Zn} \mathrm{SOD} 1)$ which destroys reactive oxygen species (ROS), but a dozen or more of other genetic mutations may also be linked to ALS. Among the primary hypotheses underlying motor neuron vulnerability are susceptibility to excitotoxicity and oxidative damage. ALS patients' spinal cord demonstrates motor neuron damages marked by cannabinoid receptor 2 (CB2) positive microglia/macrophages and cyclooxygenase-2 (COX-2) activity that fuel neuroinflammation [6,7]. Oxidative stress, mitochondrial dysfunction and excitotoxicity are thus general hallmarks.

Actual treatment options approved by the FDA are limited; two drugs received so far marketing approval, Riluzole (Rilutek ${ }^{\mathrm{rm}}$ ) and Edavarone (Radicava ${ }^{\mathrm{Tm}}$ ). Whereas Riluzole can be administered orally, Edavarone must be given by IV route. Riluzole may increase survival in certain groups by about 3 to 24 months at the best [4]; no reversal of symptoms has been reported. Complementary and alternative medicine may increase survival [2]. Given the lack of medications with a more pronounced effect on disease progression and survival, it is not surprising that many patients try complementary or alternative therapies.

\section{Case Description}

About eighteen months ago, the patient, a general practitioner at the beginning of his sixties, observed a painless weakness and impaired function of his right hand. Within about 3 weeks, paresthesia, at the beginning only on the little finger, progressed to the other fingers of the same hand, and included the foot of the same side, with a similar spread from digit 5 to digit 3, suspecting a motor neuron disease. With a slight delay, dysarthria was also observed. At the time of neurological examination, about three weeks after the first symptoms, the impaired function of the right hand and right foot as well as a slurred speech had progressed. Examination demonstrated hyporeflexia, positive Hoffmann's reflex, as well as abnormal electromyography findings with almost normal nerve conduction, suspecting ALS. There were no 
sensory abnormalities. Diagnose was made on clinical aspects only; neuroimaging was not done. No risk factor (e.g., positive family history, smoking) was identified.

Riluzole (Rilutek ${ }^{\mathrm{m}}$, Aventis Pharma S.A., Antony, France), $2 \times 50$ $\mathrm{mg} / \mathrm{d}$, was started; an increase to $2 \times 100 \mathrm{mg} / \mathrm{d}$ was not tolerated due to severe sedation. To this treatment, Sanopal ${ }^{\mathrm{m \omega}}$ (a dietary supplement consisting of alpha-ketoglutaric acid, potassium hydroxid, 5-hydroxymethylfurfural, magnesium chloride, saccharose and water, manufacturer: C.Y.L. Pharmazeutika GmbH, Laßnitzhöhe, Austria) was added. However, condition worsened, with fasciculation, muscle cramps, increased weakness, dysarthria as well as dysphagia. Therefore, 8 weeks after starting with riluzole, the patient included $2 \times$ $200 \mathrm{mg}$ cannabidiol (CBD) per day to his treatment regimen. Crystalline CBD of herbal origin (purity $>99.5 \%$ ) is available in Austria as pharmacy preparation in form of capsules, solutions of $10 \%$ and $20 \%$ or as suppositories (manufacturer: BSPG, Sandwich, UK; import: Trigal Pharma GmbH, Vienna, Austria).

Within two weeks on Co-medicationwith CBD, the patient noticed improvement of his symptoms, with a further amelioration when the dose was increased to $2 \times 300 \mathrm{mg} \mathrm{CBD} /$ day. Six weeks after starting $\mathrm{CBD}$, complete reversal of paresthesia in the foot was observed and most of paresthesia in the right hand; only a slight weakness remained in digit 5 and 4. Dysphagia also improved, whereas dysarthria remained almost stable without significant changes. Improvement was maintained for the following 10 weeks after which a slight progression of dysarthria was observed again. A dose increase to $2 \times 400 \mathrm{mg} \mathrm{CBD}$ had no additional effect. During the following 12 months (now about eighteen months after onset) dysphagia, dysarthria and fatigue progressed; speech is actually almost lost. However, other symptoms worsened much less; the patient can still use his right hand and ride his bicycle, although muscle weakness and atrophy has slightly progressed. Other functions are maintained with no significant changes.

\section{Discussion and Conclusion}

Despite of growing evidence for an implication of the endocannabinoid system in the pathophysiology of motor neuron disease including ALS, as well as of potential benefits from cannabinoids, this is, to the best of our knowledge, the first report with pure $\mathrm{CBD}$ for treating a motor neuron disease.

Preclinical studies suggest neuroprotective and antiapoptotic activities of cannabinoids in neurodegenerative processes whereby animal experiments using a transgenic ALS mouse model demonstrated a prolongation of survival with Delta-9tetrahydrocannabinol [8]. Later, a combination of phytocannabinoids, THC and CBD in a ratio of about 1:1 (nabiximols/Sativex $\left.{ }^{\mathrm{rn}}\right)$, was tested in SOD1-G93A mice with moderately positive results [9]. In two anonymous surveys, each including over 100 ALS patients, $10 \%$ to $21 \%$ judged medical marijuana very effective, particularly in stimulating appetite (75\%), aiding sleep (65\%), relieving anxiety (80\%), relieving depression $(70 \%)$, and providing muscle relaxation $(60 \%)[10,11]$. Annecdotal community reports exist as well. Further on, a four weeks, randomized, double-blind, cross-over pilot study of 19 ALS patients, 2.5-10 mg of dronabinol (synthetic THC) per day was associated with improvements in sleep and appetite; however cramps or fasciculation did not improve. A dose of $10 \mathrm{mg}$ THC is considered dose-limiting [12]. These few clinical studies with medical marijuana, extracts or dronabinol demonstrate alleviation of some ALS-related symptoms; however, control of disease progression or even reversal was not reported. As up to one half of the motor neurons innervating a muscle may be lost in ALS before clinical signs of weakness or atrophy are found [13], rapid diagnosis and early onset of treatment seems to be crucial to delay symptom development.

CBD, a non-psychotropic cannabinoid, is well known for its multitarget effects, with potent anti-inflammatory and neuro-protective properties in neurological preclinical models [14], although the antiinflammatory mechanism is still incompletely understood. Experiments have demonstrated that low doses of CBD act as adenosine A2A receptor-and Peroxisome Proliferator Activated Receptor gamma (PPARg) agonist, and as G-protein coupled receptor 55 (GPR55) antagonist, decreasing the levels of inflammatory mediators such as TNF-alpha, IL-6 and IL-12, without acting directly on cyclooxygenase 1 or 2 (COX-1, COX-2 [15]. In addition, CBD is a very potent anti-oxidant, more protective than $\alpha$-tocopherol or vitamin $\mathrm{C}$ [16]. On CB1 receptors, $\mathrm{CBD}$ acts as negative allosterical modulator, decreasing partially the activity of ligands [17]. On CB2 receptors, $\mathrm{CBD}$ can act as receptor-inverse agonist. This may explain, at least in part, its anti-inflammatory effects and its inhibition of the migration of macrophages, microglial cells and neutrophils [18]. CBD shares with Riluzole effects on ion channels that are responsible for repetitive firing and transmitter release [19]. As some cases seem to be associated with mutations in the gene for copper-zinc superoxide dismutase $1(\mathrm{Cu}-\mathrm{Zn}$ SOD1), a powerful natural antioxidant, it is noteworthy that CBD alters the expression of a wide range of genes involved in zinc homeostasis, oxidative stress, mitochondrial dysfunction, excitotoxicity, glutathione deprivation and anti-inflammatory signalling pathways $[20,21]$.

These mechanisms of CBD are interesting as they fit many of the current hypothetical requirements for a successful drug for motor neuron diseases. Further on, CBD seems to be well tolerated and safe in humans, even at high doses and with chronic use [22], and can easily be combined with existing treatment regimens. However, more observations and thorough investigations are needed for assessing the potential role of $\mathrm{CBD}$ in this deadly disease.

\section{References}

1. Teixeira AG, Silva DL, Oliveira KS, Santana FM, Matias GDM, et al. (2015) Amyotrophic lateral sclerosis: The current world situation. Int Arch Med 8: 1-8.

2. Bedlack RS, Joyce N, Carter GT, Pagononi S, Karam C (2015) Complementary and alternative therapies in ALS. Neurol Clin 33: 909-936.

3. Cetin H, Rath J, Fuzi J, Reichardt B, Fülöp G, et al. (2015) Epidemiology of amyotrophic lateral sclerosis and effect of riluzole on disease course. Neuroepidemiology 44: 6-15.

4. Chen L, Liu X, Tang L, Zhang N, Fan D (2016) Long-term use of riluzole could improve the prognosis of sporadic amyotrophic lateral sclerosis patients: A real-world cohort study in China. Front Aging Neurosci 8: 246.

5. Chen L, Zhang B, Chen R, TangL, Liu R, et al. (2015) Natural history and clinical features of sporadic amyotrophic lateral sclerosis in China. J Neurol Neurosurg Psychiatry 86: 1075-1081.

6. Di Iorio G, Lupi M, Sarchione F, Matarazzo I, Santacroce R, et al. (2013) The endocannabinoid system: A putative role in neurodegenerative diseases. Int J High Risk Behav Addict 2: 100-106.

7. Yiangou Y, Facer P, Durrenberger P, Chessell IP, Naylor A, et al. (2006) COX-2, CB2 and P2X7-immunoreactivities are increased in activated microglial cells/macrophages of multiple sclerosis and amyotrophic lateral sclerosis spinal cord. BMC Neurol 6: 12. 
Citation: Nahler G (2017) Co-medication with Cannabidiol May Slow Down the Progression of Motor Neuron Disease: A Case Report. J Gen

8. Raman C, McAllister SD, Rizvi G, Patel SG, Moore DH, et al. (2004) Amyotrophic lateral sclerosis: Delayed disease progression in mice by treatment with a cannabinoid. Amyotrophic Lateral Scler Other Motor Neuron Disord 5: 33-39.

9. Martet MM, Porras EF, Ruiz FJ, de Lago E (2014) Changes in endocannabinoid receptors and enzymes in the spinal cord of SOD1(G93A) transgenic mice and evaluation of a Sativex( $\left(^{\circledast}\right)$-like combination of phytocannabinoids: Interest for future therapies in amyotrophic lateral sclerosis. CNS Neurosci Ther 20: 809-815.

10. Amtmann D, Weydt P, Johnson KL, Jensen MP, Carter GT (2004) Survey of cannabis use in patients with amyotrophic lateral sclerosis. Am J Hosp Palliat Care 21: 95-104.

11. Kaufman J, Almasy K, Boller A, Dahodwala N, Elman L, et al. Medical marijuana utilization and perceived therapeutic value in patients with ALS. Neurology 82 : P3.014.

12. Joerger M, Wikins J, Fagagnini S, Baldinger R, Brenneisen R, et al. (2012) Single-dose pharmacokinetics and tolerability of oral delta-9tetrahydrocannabinol in patients with amyotrophic lateral sclerosis. Drug Metab Lett 6: 102-108.

13. Daube JR (2000) Electrodiagnostic studies in ALS and other motor neuron disorders. Muscle Nerve 23: 1488-1502.

14. Bih CI, Chen T, Nunn AVM, Bazelot M, Dallas M, et al. (2015) Molecular targets of cannabidiol in neurological disorders. Neurotherapeutics 12: 699-730.
15. Burstein S (2015) Cannabidiol (CBD) and its analogs: A review of their effects on inflammation. Bioorg Med Chem 23: 1377-1385.

16. Hampson AJ, Grimaldi M, Lolic M, Wink D, Rosenthal R, et al. (2000) Neuroprotective antioxidants from marijuana. Ann NY Acad Sci 899: 274-282.

17. Laprairie RB, Bagher AM, Kelly ME, Wright DEM (2015) Cannabidiol is a negative allosteric modulator of the type 1 cannabinoid receptor. $\mathrm{Br} \mathrm{J}$ Pharmacol 172: 4790-4805.

18. Pisanti S, Malfitano AM, Ciaglia E, Lamberti A, Ranieri R, et al. (2017) Cannabidiol: State of the art and new challenges for therapeutic applications. Pharmacol Ther 175: 133-150.

19. Bellingham MC (2011) A review of the neural mechanisms of action and clinical efficiency of riluzole in treating amyotrophic lateral sclerosis: What have we learned in the last decade? CNS Neurosci Ther 17: 4-31.

20. Rajan TS, Scionti D, Diomede F, Grassi G, Pollastro F, et al. (2017) Cannabidiol modulates genes linked with amyotrophic lateral sclerosis. J Cell Biochem 118: 819-828.

21. Juknat A, Rimmermann N, Levy R, Vogel Z, Kozela E (2012) Cannabidiol affects the expression of genes involved in zinc homeostasis in BV-2 microglial cells. Neurochemistry Int 61: 923-930.

22. Bergamaschi MM, Queiroz RH, Crippa JAS, Zuardi AW (2011) Safety and side effects of cannabidiol, a Cannabis sativa constituent. Curr Drug Safety 6: 237-249. 